\title{
Investigation of Particulate Flow in a Channel by Application of CFD, DEM and LDA/PDA
}

\author{
M. Azimian, M. Lichti and H.-J. Bart* \\ Chair of Separation Science and Technology, University of Kaiserslautern, D-67663 Kaiserslautern, Germany
}

\begin{abstract}
Hydroabrasion in particulate flows plays an important role in various industrial and natural processes. To predict the influence of it in a pipeline, channel or a fitting, it is essential to characterize the effects in a simple standardized geometry. An example to this is a pipe channel with a cylindrical obstacle adjusted inside the channel perpendicular to the flow direction. Results of flow field are generated by using the non-invasive Laser/Phase Doppler Anemometry (LDA/PDA) measurement technique. The velocity profiles of single phase and particulate flow from computational fluid dynamics (CFD) and discrete element method (DEM) simulations were validated by the LDA experimental data. The simulations were performed on the basis of Euler-Lagrange technique for both CFD and DEM. The measurements show that a Karman vortex field forms behind the obstacle and particles move inside this field with an average negative velocity of up to $25 \%$ of the fully developed velocity field. A comparison of CFD and DEM results with experimental data showed that in Karman velocity field, the CFD results fit better to the LDA measurements. In the fully developed flow region and also above and under the vortex field behind the obstacle, the DEM results match better with the LDA data.
\end{abstract}

Keywords: CFD, cylindrical channel, DEM, hydroabrasion, LDA/PDA.

\section{INTRODUCTION}

Particulate flows are responsible for accelerated abrasion in pipelines and plant equipment which significantly shortens the service life and increase the repair and maintenance costs and is an important factor which should be considered in a sustainable design. The existing literature mainly covers LDA and Particle Image Velocimetry (PIV) measurements of gas-liquid multiphase flow systems with very few references on solid particles-liquid flows. To our best knowledge, data on velocity profiles of solid particles and the deviation compared to the liquid phase, velocity fluctuations and the particles size distribution has not been measured with LDA/PDA technique for solid particles-liquid flows with sufficient detail and accuracy.

Sommerfeld [1] reviewed a series of experimental works concerning the achievement of a detailed analysis of solid particles entrained in a gas flow in pipes and channels. Huber and Sommerfeld [2] applied the PDA and a laser light sheet technique to measure the solid particles concentration in a pipe along the cross section. Moreover, a very detailed set of experiments was provided by Tsuji and Morikawa [3] for a gas-solid flow in a horizontal pipe using various types of relatively large spherical particles. Ozgoren et al. [4] applied PIV to investigate and compare the flow structures in the downstream region of a cylindrical and spherical obstacle fixed in a channel flow. Kumara et al. [5] has compared the PIV and LDA measurement techniques applied to the oilwater two phase flow in a horizontal pipe. Ristic et al. [6]

*Address correspondence to this author at the Chair of Separation Science and Technology, University of Kaiserslautern, D-67663 Kaiserslautern, Germany; Tel: +49 (0)631 205-2414; Fax: +49 (0)631 205-2119;

E-mail: bart@mv.uni-kl.de presented an overview of uncertainty sources in the LDA measurements. He estimated the LDA measuring volume positioning in cylindrical pipe flow due to optical aberrations caused by the pipe wall curvature. Durst et al. [7] applied the LDA method to estimate the local wall shear stress from near-wall mean velocity data in fully developed pipe and channel flows. He validated the direct numerical simulation (DNS) results with the LDA data.

The purpose of this paper is to investigate the solid particles motion and interactions in a pipe with application of an optical non-invasive measurement technique in order to better understand the solid particles behavior in liquid flow. In that respect, LDA/PDA measurements were used to validate the results gained by application of CFD and DEM simulation techniques. Furthermore, an erosion model derived from experimental results in pipe parts and chokes was implemented in the CFD tool to predict accurately the local material loss and erosion profile on the obstacle surface in the pipe. The goal of the accurate erosion modeling is to strengthen the instrument in locations where the erosion occurs and perform further optimization of a given geometry to minimize the local erosion rate.

\section{EXPERIMENTAL SETUP}

A closed loop experimental tester as presented in (Fig. 1) was built to perform the LDA/PDA measurements in a cylindrical channel with an obstacle fixed inside it perpendicular to the flow direction. A slurry centrifugal pump is used to pump a diluted mixture of water and sand particles from a mixing tank to the cylindrical channel. The obstacle is placed near to the outlet part of the cylindrical channel in order to have a long entering length of over 20 times the channel diameter. This results the flow to have a fully developed veloc- 


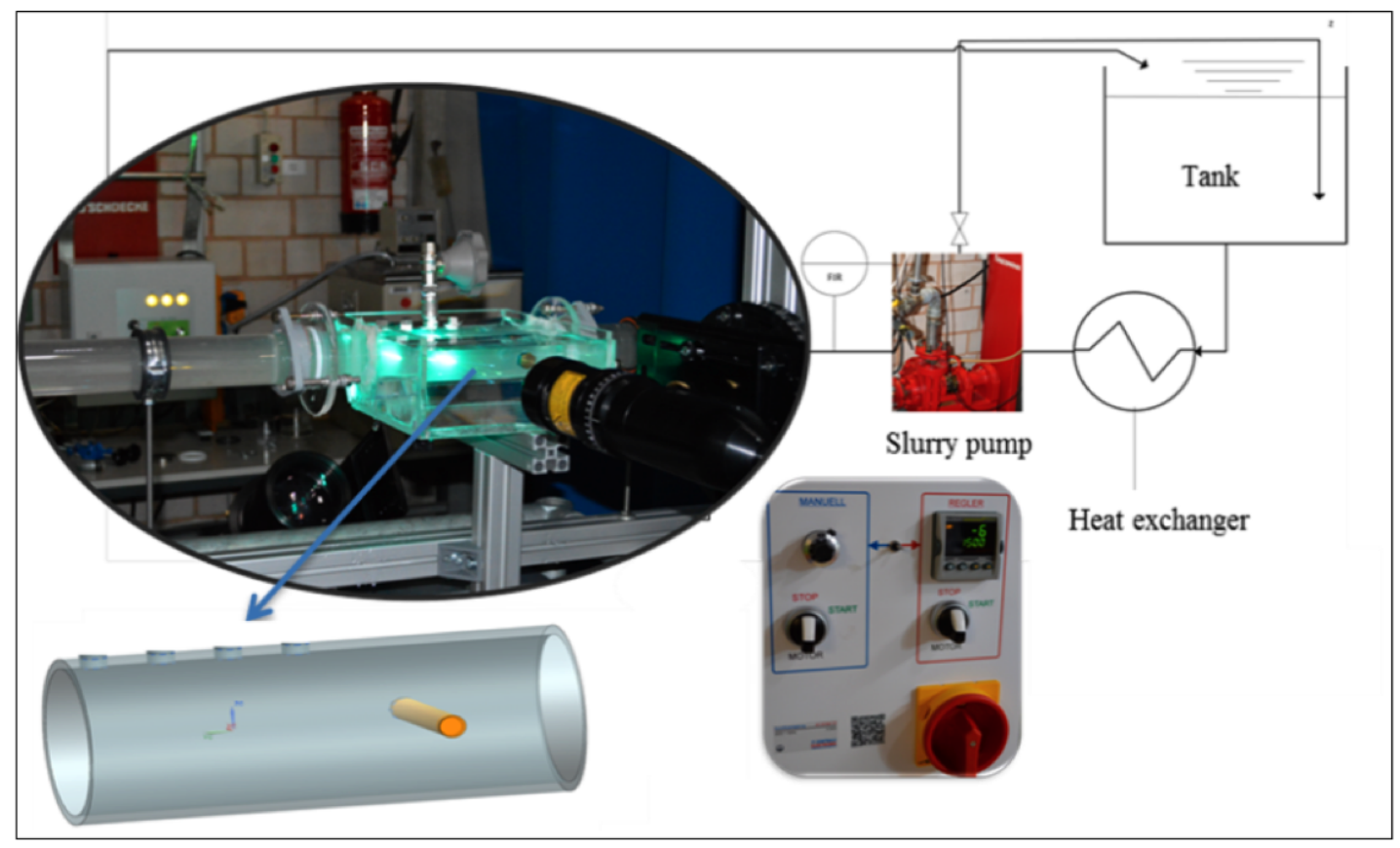

Fig. (1). LDA/PDA closed loop experimental tester.

ity profile before facing the obstacle. The fixed obstacle inside the channel has a cylindrical shape but can be replaced with any other geometry for further studies. A bypass flow guides a high amount of flow back to the mixing tank in order to provide a homogeneous mixture of solid particles and fluid phase. A heat exchanger is used to keep the mixture at constant temperature of $20{ }^{\circ} \mathrm{C}$. As depicted in Fig. (1), a regulator is used to provide various volumetric feed flows. A box made from para-glass was built in the measurement section around the cylindrical channel which was filled with deionized water and used to minimize the laser beams deflections. LDA/PDA is used to measure the solid particles and fluid velocity profiles before and after the obstacle at various defined positions in order to compare with each other and also to validate the results gained by ANSYS-CFX and EDEM-FLUENT simulations.

The LDA method is applied in present work to measure the velocity profiles of the single phase and the particulate flow. The light source of the LDA is a water-cooled (up to 4 W) Coherent Innova $70 \mathrm{C}$ Argon Ion Laser which produces a coherent laser beam. The single laser beam split into two parts by two prisms when the beam enters the splitter. The beams are then lead into the Bragg cell and followed afterwards to the four beam module. The shifted and non-shifted beams enter the module where they split into two blue and two green beams. Each beam pair contains one shifted beam and one non-shifted beam and has different wavelengths, blue has $488 \mathrm{~nm}$ and green has $514.5 \mathrm{~nm}$. The laser beams are directed into transmitting fiber optical cords that are connected to the transmitting probe. When the system is properly aligned, each individual beam has the same power. An optical power-meter was used to measure the beams intensity and the maximum power was up to $40 \mathrm{~mW}$ for each beam coming out from the probes. The front lens with focal length of $250 \mathrm{~mm}$ focuses the four beams at the focal point. The detector is a photomultiplier tube (PMT) which converts the light into electrical data that are sent to the signal processor. The signal processor is then connected to a computer. The optical system was mounted on a 3-axis traversing bench (Isel) which could position the measurement volume with a resolution of $0.01 \mathrm{~mm}$. It allows measurements of local velocities across the pipe diameter to produce the entire velocity profiles at various cross sectional positions [5]. The designed software of Flowsizer 3.0 by TSI processes the raw data into statistical values like root mean squared (rms) values of velocity fluctuations, mean velocities, and Reynolds stress.

For measuring the velocity profiles of the single phase flow, first of all, the appropriate tracer particles should be selected in order to represent the same liquid flow field characteristics. Particle size, composition, density, shape and concentration are important factors when selecting tracer particles. The ability of a particle to follow fluctuations in the flow depends on the particle's aerodynamic diameter $d_{a}$ which depends on the particle's geometric diameter $d_{g}$ and density $\rho$ according to the following equation:

$$
d_{a}=d_{g} \cdot \sqrt{\rho}
$$

A smaller aerodynamic diameter is associated with a higher frequency response and a greater ability to follow rapid flow fluctuations. The tracer particles must be small enough to follow the flow faithfully but also big enough to scatter sufficient light for signal detection. For the current study, hollow glass sphere particles with nominal mean diameter of 8 to $12 \mu m$ (Potters spherical $110 \mathrm{p} 8$ ) and refractive index of 1.5 were selected as tracer particles. As abrasive particles, two groups of sand particles with different size distributions as presented in (Fig. 2) are applied. The sand particle size distribution is meaured by a HORIBA particle size analyzer (Retsch Technology, LA950). The mentioned sand particles size ranges $100-200 \mu \mathrm{m}$ and 200 - 
$315 \mu \mathrm{m}$ refer to the filter plates that were used to filter the particles with the vibration filtering method. Since many of sand particles are not spherical, there might be smaller or larger particles present in the filtered particles collected as presented in (Fig. 2).

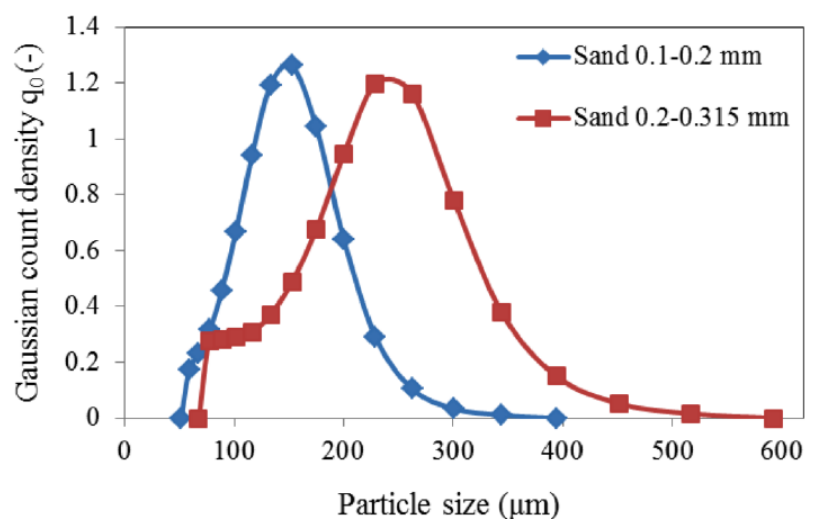

Fig. (2). Sand particles size distributions used in exp. measurements.

\section{DISCRETE ELEMENT METHOD (DEM)}

Discrete or distinct element method (DEM) is a family of numerical methods for computing the motion and effects of a large number of particles. The most important advantage of the DEM is that complex systems can be modeled with basic data without over simplifying assumptions. For example, in the transition from non-moving to moving particles or in mixing and segregation problems. The main limitation of DEM is the computational requirement, since the method inherently uses an explicit time integration scheme and repeats sequential calculations over a limited time period with very small time steps. However, due to increase in computational capacity, DEM became a popular computational tool. Moreover, the number of particles that can be reasonably simulated is still limited and the product of number of particles and number of time steps should be considered [8]. Detailed basic principles of DEM simulations can be found in the recent review of Zhu et al. [9].

The basic methodology of DEM for a dynamic Newtonian analysis is outlined as follows which is given by Asmar et al. [10] in detail:

a) Search the system and reference all particles.

b) Apply interaction laws (calculating forces and moments) over all particle-particle and particle-wall contacts.

c) Apply other influencing forces such as gravity, pressure gradient, lift force and fluid drag to each particle.

d) Apply Newton's second law to determine each particle's translational and rotational acceleration from the affecting forces and moments.

e) Use the numerical integration to update particle positions and velocities at the next time step.

Applying the discrete element method, the trajectory of each particle is computed to simulate the behavior of the whole particulate flow. At every time step the forces acting on each particle are updated and Newton's equation of motions are solved to obtain the new positions and velocities of the particles. The forces acting on the particles include the contact forces between the particles as well as additional cohesion forces that also act between two particles but are not connected necessarily with a physical contact of the two particles. The total momentum acting on the particle has to be taken into account which is evaluated from the contribution of each individual force by multiplication with the normal distance with respect to the center of gravity for each individual particle [11].

\section{CFD-DEM MODEL}

The fluid-phase (continuum) dynamics are described by the incompressible Newtonian fluid based on an Euler approach. In contrast, the dispersed phase or the particles are treated as a collection on individual particles, whose movement is described by applying Newton's second law. Thus, the model is based on an Euler-Lagrange approach for a non-reactive flow. The continuum and dispersed phases are strongly coupled via the momentum exchange between fluid phase and particles. Particles collisions are also important, and therefore must be taken into account by DEM. In the following sections, the particle phase and the fluid phase equations are presented, followed by a description of the input data for DEM simulations used in the present work.

\subsection{Fluid Phase}

The motion of the fluid phase is calculated from the volume averaged fluid phase governing equations as given by Jackson [12]. The continuity equation for the fluid phase is given by equation (2) and the momentum equation by equation (3):

$$
\begin{aligned}
& \frac{\partial\left(\rho_{f} \varepsilon_{f}\right)}{\partial t}+\nabla \cdot\left(\rho_{f} \varepsilon_{f} u_{f}\right)=0 \\
& \rho_{f} \varepsilon_{f}\left[\frac{\partial u_{f}}{\partial t}+\left(u_{f} \cdot \nabla\right) u_{f}\right]=-\varepsilon_{f} \nabla P+\varepsilon_{f} \nabla \cdot \tau_{f}-\varepsilon_{f} M
\end{aligned}
$$

where $\mathrm{M}$ is the interphase momentum transfer between the fluid phase and each individual particle, $\nabla P$ is the static pressure gradient and $\nabla \cdot \tau_{f}$ is the viscous force where the term $\tau_{f}$ is the stress tensor for fluid phase.

\subsection{Particle Phase}

The solution of the equation of motion for each computational particle is essential for the simulation of the solid particle phase by the Lagrangian approach. This equation includes the particle inertia, drag, gravity buoyancy, slip shear lift force and slip rotational lift force. The change of the angular velocity along the particle trajectory results from viscous interaction with the fluid (torque $\vec{T}$ ) and wall collisions [13]. Thereby, the equations of motion for the solid particles are given as follows:

$$
\begin{aligned}
m_{p} \frac{d}{d t}\left(\frac{d x_{p i}}{d t}\right) & =\frac{3}{4} \frac{\rho}{\rho_{p} D_{p}} m_{p} C_{D}\left(u_{i}-u_{p i}\right)\left|\vec{u}-\vec{u}_{p}\right| \\
& +m_{p} g_{i}\left(1-\frac{\rho}{\rho_{p}}\right)+F_{r i}+F_{s i}
\end{aligned}
$$




$$
I_{p} \frac{d \omega_{p i}}{d t}=T_{i}
$$

Here, $x_{p i}$ is the coordinate of particle position, $u_{p i}$ is its velocity component, $u_{i}=U_{i}+u_{i}^{\prime}$ is the component of the instantaneous velocity of the fluid, $D_{p}$ and $\rho_{p}$ are the particle diameter and density, respectively. $m_{p}=(\pi / 6) \rho_{p} D_{p}^{3}$ is the particle mass and $I_{p}=0.1 m_{p} D_{p}^{2}$ is the moment of inertia of a sphere. Using the standard correlation, the drag coefficient is obtained as:

$$
C_{D}=\left\{\begin{array}{cc}
24 \mathrm{Re}_{p}^{-1}\left(1+0.15 \mathrm{Re}_{p}^{0.687}\right) & \mathrm{Re}_{\mathrm{p}} \leq 1000 \\
0.44 & \mathrm{Re}_{\mathrm{p}}>1000
\end{array}\right.
$$

where $\operatorname{Re}_{p}=\rho D_{p}\left|\vec{u}-\vec{u}_{p}\right| / \mu$ is the particle Reynolds number. The slip shear force is based on the analytical result of Saffman [14] and developed for higher particle Reynolds numbers by Mei [15]:

$$
\vec{F}_{s}=1.615 D_{p} \mu \operatorname{Re}_{s}^{0.5} C_{s} \frac{\left[\left(\vec{u}-\vec{u}_{p}\right) \times \vec{\omega}\right]}{|\vec{\omega}|}
$$

where $\operatorname{Re}_{s}=\rho D_{p}^{2}|\vec{\omega}| / \mu$ is the particle Reynolds number of the shear flow, $\vec{\omega}=\nabla \times \vec{u}$ is the fluid rotation and $C_{s}=F_{s} / F_{s, \text { Saff }}$ represents the ratio of the extended lift force to the Saffman force:

$$
C_{s}= \begin{cases}\left(1-0.3314 \beta^{0.5}\right) e^{-\mathrm{Re}_{p} / 10}+0.3314 \beta^{0.5} & \operatorname{Re}_{\mathrm{p}} \leq 40 \\ 0.0524\left(\beta \cdot \operatorname{Re}_{\mathrm{p}}\right)^{0.5} & \operatorname{Re}_{\mathrm{p}}>40\end{cases}
$$

where $\beta$ is a parameter given by: $\beta=0.5 \operatorname{Re}_{s} / \operatorname{Re}_{p}(0.005<\beta<0.4)$.

The applied slip rotational lift force was extended to account for the relative motion between fluid and particle and is in the following form [16]:

$$
\vec{F}_{r}=\frac{\pi}{8} D_{p}^{3} \rho \frac{\operatorname{Re}_{p}}{\operatorname{Re}_{r}} C_{r}\left[\vec{\Omega} \times\left(\vec{u}-\vec{u}_{p}\right)\right]
$$

where $\vec{\Omega}$ is defined as $\vec{\Omega}=0.5\left(\vec{\omega}-\vec{\omega}_{p}\right)$ and the Reynolds number of particle rotation is given by $\operatorname{Re}_{r}=\rho D_{p}^{2}|\vec{\Omega}| / \mu$. The lift coefficient for $\operatorname{Re}_{p}<2000$ could be taken from experimental work of Oesterle and Dinh [16].

\section{SIMULATION OF SOLID PARTICLES BY EDEM- FLUENT COUPLING}

In the present study the ANSYS-FLUENT was coupled with EDEM (DEM Solutions) software to investigate the solid particles motion and comparison with LDA data and also the results gained by CFX simulations. For this purpose, the coupling module (open source project) was adapted with the FLUENT version 14.0 and was recompiled again. First, a potent turbulent fully developed velocity profile as a user defined function (UDF) in C code was given for the inlet velocity boundary condition. The inlet velocity profile was later modified by creating a fitted function for the velocity profile on the basis of LDA measured values to reflect the reality better. The modified profile is based on the equation of Michaelis-Menten kinetics (10). The two parameters in equation (11) are adjusted to experimental values so that the error amount was minimal. The variable $r$ is the pipe radius, the $\mathrm{c}_{\max }$ is the maximum velocity of the profile and $\mathrm{k}_{2}$ is a constant to fit the velocity profile to the LDA measured data. In order to represent the equation for different volumetric feed flows, a factor $\mathrm{K}_{1}$ is necessary, which was determined by numerical integration of the profile. For $\mathrm{r}:[-\mathrm{R}: 0]$ and the mean velocity of $\overline{\mathrm{c}}$, the equation of velocity profile in a cylindrical channel is derived as equation (11).

$$
\begin{aligned}
& c(r)=\frac{\mathrm{c}_{\text {max }} r}{\mathrm{~K}_{2}+r} \\
& c(r)=K_{1}(\overline{\mathrm{c}}) \cdot \frac{\left.\mathrm{c}_{\text {max }} \cdot\left(1-\mid \frac{r}{R}\right)\right)}{\mathrm{K}_{2}+\left(1-\left|\frac{r}{R}\right|\right)}
\end{aligned}
$$

Initial simulations were performed by a two way coupling between ANSYS-FLUENT and EDEM, it means in every time step the result from EDEM was transferred to FLUENT and there, the effects of particles on fluid flow were considered, the fluid flow result after solution convergence was again transferred to EDEM for the next time step simulation and to consider the effects of fluid flow on solid particles. Fig. (3) presents a two way coupled simulation between EDEM and FLUENT with a grid having around 1.5 million elements and is gained after three weeks of simulation run. In order to reduce the computational time, the one way coupling between the FLUENT and EDEM was implemented and tested. This is done by importing the calculated fluid flow and vortex field from FLUENT into EDEM and there used as the basis for the calculation of influencing forces like lift and drag forces affecting the particles. Basic assumption is that the flow field is only slightly affected by the particles in a relatively dilute suspension. The parameters, especially the interactions between the particles or particle-surface interactions (restitution coefficient and friction coefficient) for applying in the DEM simulation should be estimated according to the literature experimental data. Furthermore, the models which predict the various affecting forces on the particles need to be validated. The Rayleigh time step is also an important parameter which should be calculated and set into the DEM simulations. It is a theoretical maximum time step for a DEM simulation of a quasi-static particulate collective in which the total number of contacts per particle remains above 1 . The time step for the DEM simulations is calculated with the following equation:

$$
t_{R}=\pi R\left(\frac{\rho}{G}\right)^{\frac{1}{2}} /(0.1631 \cdot v+0.8766)
$$

where $v$ is the Poisson's ratio, $\mathrm{G}$ is the shear modulus, $\mathrm{R}$ and $\rho$ are the particle's radius and density, respectively. For the current DEM simulations, the time step was calculated as about $2 \cdot 10^{-8} \mathrm{~s}$.

Beside the FLUENT-EDEM simulations, pure CFD simulations applying the ANSYS-CFX tool were performed to predict the tracer and solid particles velocity profiles in cylindrical channel before and after the obstacle at various positions and also to investigate the material loss and erosion profile on the obstacle surface in different operating conditions by dense suspension flows. The particles streamlines over the cylindrical obstacle simulated by the CFX tool are depicted as an example in (Fig. 4). 


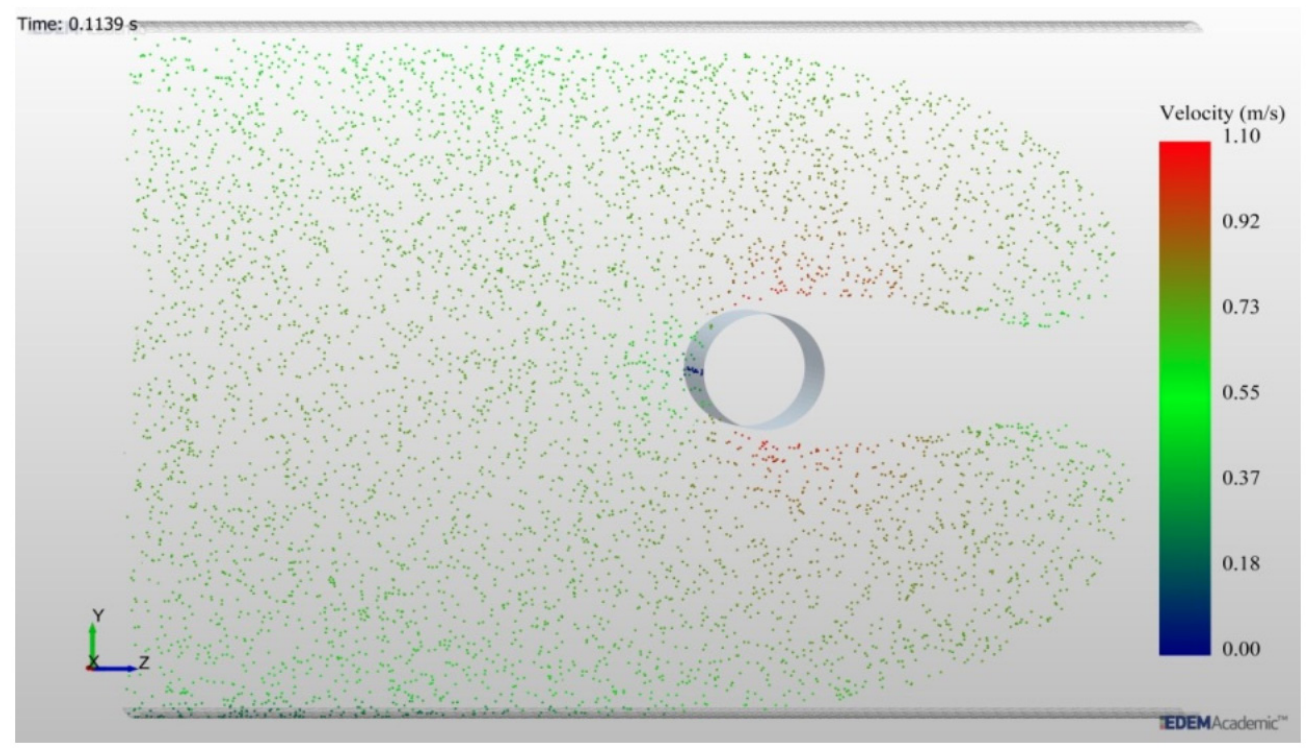

Fig. (3). Sand particles simulation with two way EDEM and FLUENT coupling.

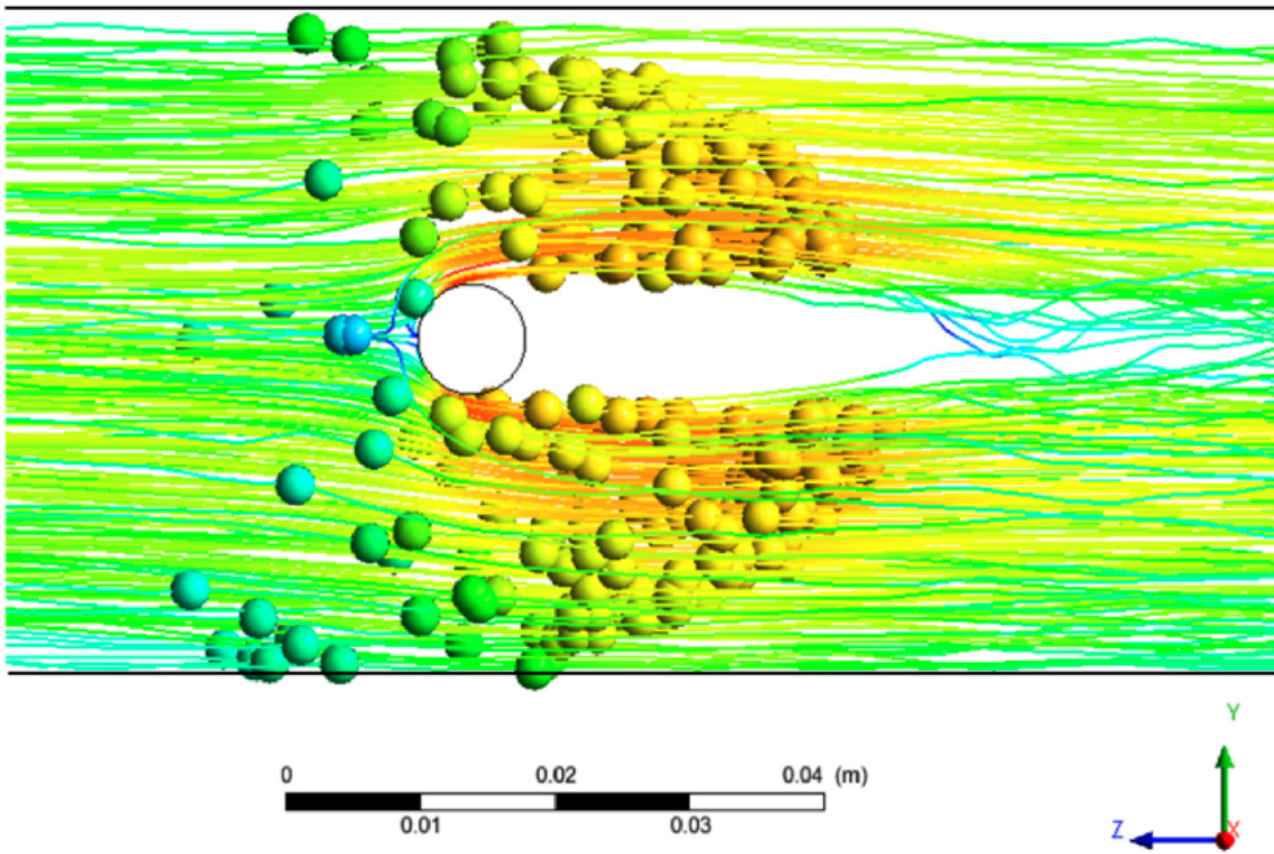

Fig. (4). Sand particles tracking with ANSYS-CFX.

In Fig. (5), velocity profiles of tracer particles at position of $34 \mathrm{~mm}$ before the flow reaches the obstacle, for five various volumetric feed flows from 3000 to $5000 \mathrm{l} / \mathrm{h}$ are presented. Connecting lines in this figure are brought in order to better visualize the variation of velocity profiles at top and bottom of the pipe cross section.

Tracer particles have hardly any influence on the flow behavior of the fluid due to their small size and identical density as the water. The applied flow rate is linearly proportional to the measured velocity of the tracer particles. It was found that average velocities above the center of the tube are slightly larger than the bottom half of the pipe cross section. This coincides with the results of previous experiments by Roco and Shook [17] which express that the lower particle concentration at upper part compared to the lower region is the main reason for the relatively larger velocities at the upper part.

The velocity profile of solid particles with the volumetric feed flow of $4000 \mathrm{l} / \mathrm{h}$ at a position of $34 \mathrm{~mm}$ before the flow reaches the obstacle are measured by LDA for over 65 points from bottom of the pipe cross section till the top point. The results obtained from CFX simulation and coupled EDEMFLUENT simulation are compared with the LDA measured data and presented in (Fig. 6). In the fully developed velocity field as depicted, except at the top of the pipe cross section, at other points, the DEM-FLUENT coupled results match better with the LDA measurement data. The results obtained from EDEM-FLUENT coupling are presented in all figures named simply as DEM. The reason for the fluctuating DEM results at top of the cross section should be further 


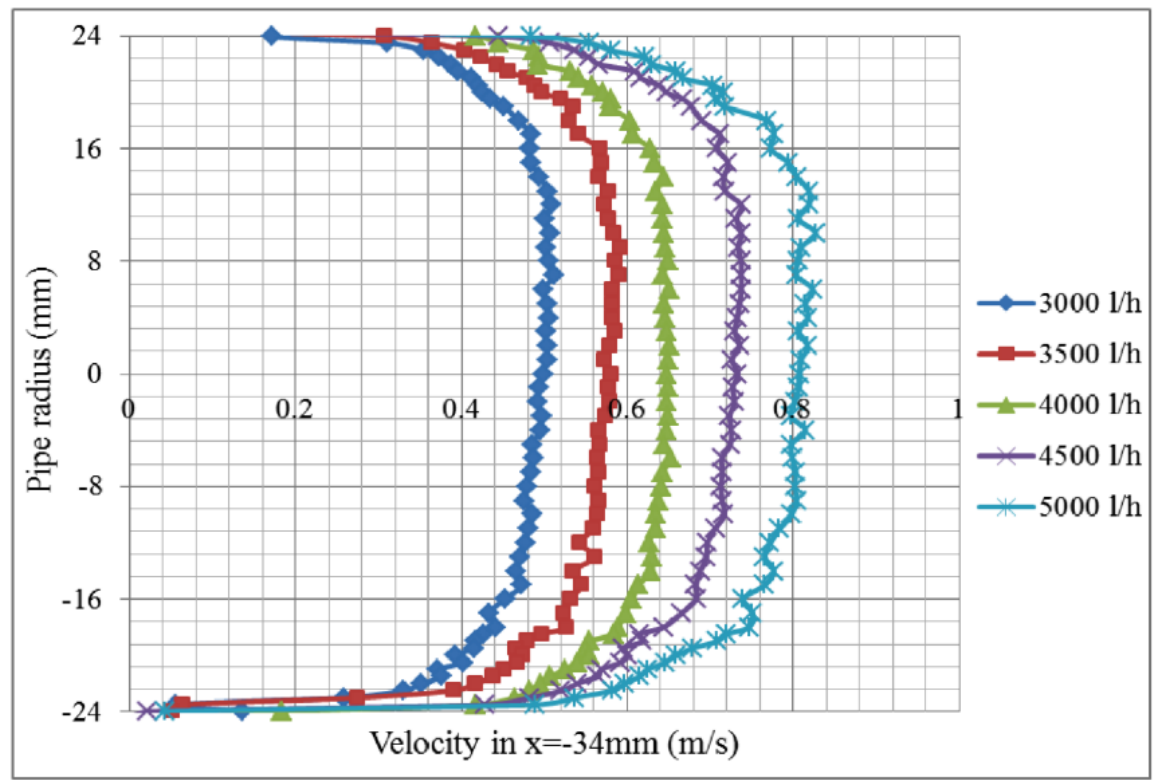

Fig. (5). Tracer particles velocity profiles by LDA for various feed flow rates at $x=-34 \mathrm{~mm}$.

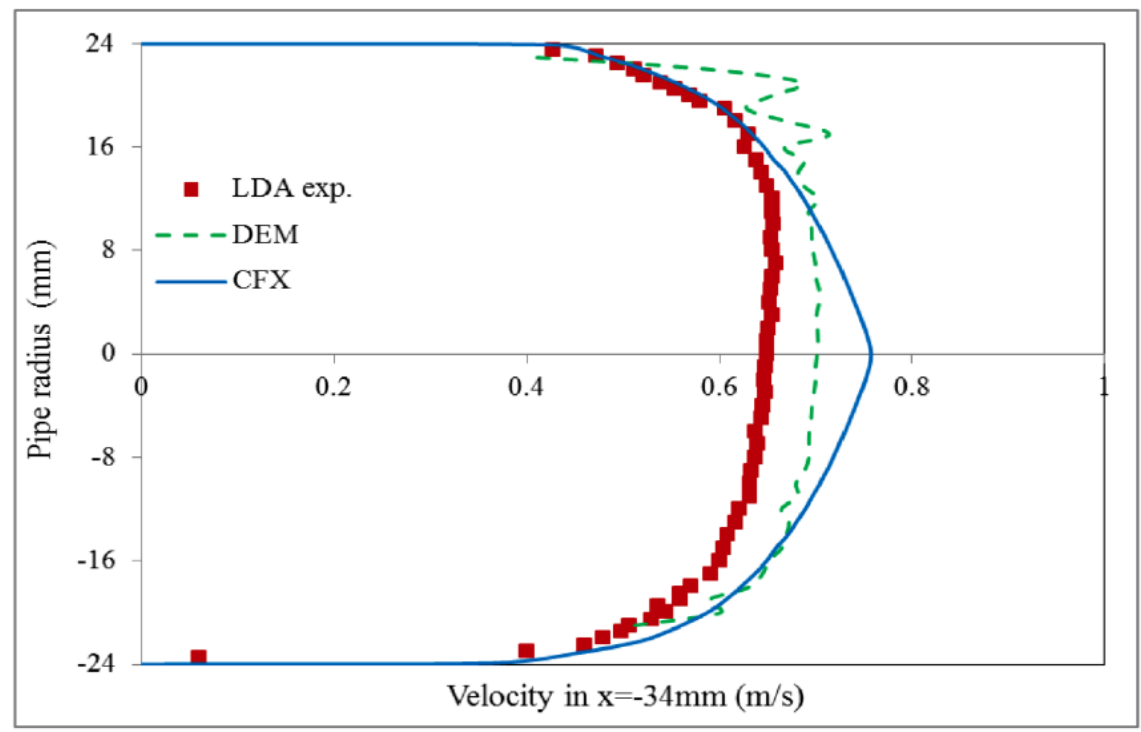

Fig. (6). Sand particles velocity profile at position of $34 \mathrm{~mm}$ before the obstacle.

investigated by increasing the particle numbers and also further grid refinements. The tracer and also sand particles velocity profiles after the suspension reached the obstacle in the flow direction were also measured at various distances from the obstacle and used for validation of CFX and DEM results. In Fig. (7), the velocity profile and its variation for different feed volumetric flows from 3000 to $5000 \mathrm{l} / \mathrm{h}$ at a distance of $16 \mathrm{~mm}$ behind the obstacle are presented.

As it is depicted, due to high turbulence intensity behind the cylindrical obstacle, the tracer particles reach negative velocities up to $25 \%$ of the maximum velocity in the Karman vortex field as compared to the fully developed velocity field. Moreover, the velocity profiles of the tracer particles at various distances before and after the obstacle in flow direction are depicted in (Fig. 8) and the redeveloping of the velocity profile behind the obstacle as the distance from the obstacle increases was observed.
A comparison among the velocity profiles of tracer particles in the size range of $8-12 \mu \mathrm{m}$, sand particles in the size range of 100-200 $\mu \mathrm{m}$ and also in the size range of 200-315 $\mu \mathrm{m}$ were done and presented in (Fig. 9). With respect to the measured profiles, there is a little variation when the particles are added to the fluid flow. Compared to the singlephase flow, the sand particles have a slightly lower average velocity. The vortex field is slightly wider, which is due to the increased shear forces of the solid particles.

As depicted in Fig. (10), the sand particles size distribution is measured by PDA. As it is obsevred it is qualitatively in good agreement with the sand size distribution presented in (Fig. 2). Furthermore, the DEM and CFX results have been compared and validated with the LDA experimental data for three various positions of $16 \mathrm{~mm}$, $28 \mathrm{~mm}$ and $48 \mathrm{~mm}$ distanced from the obstacle and brought in (Fig. 11). The comparison showed that in Karman vortex 


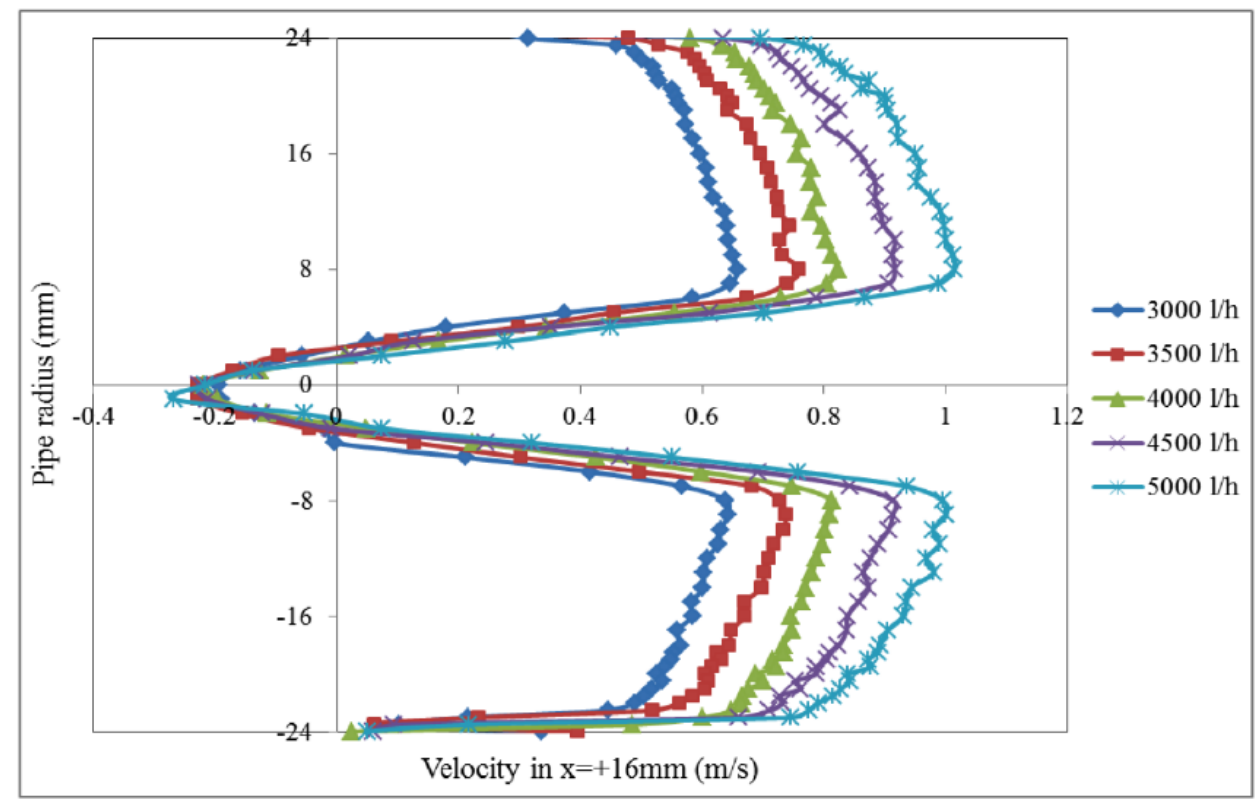

Fig. (7). Tracer particles velocity profiles by LDA for various feed flow rates at $x=+16 \mathrm{~mm}$.

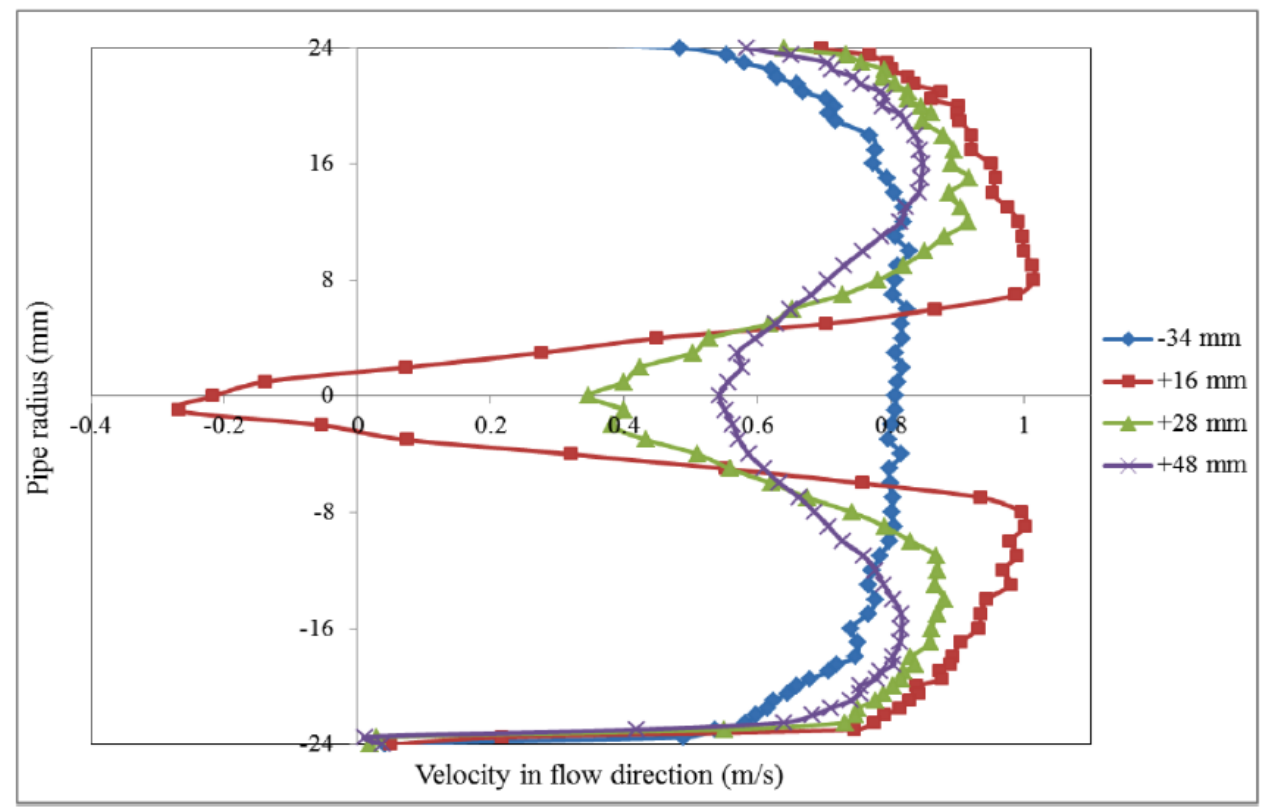

Fig. (8). Tracer particles vel. profiles at various distances from the obstacle at V=5000 1/h.

field, the CFD results fit better to the LDA measurements and above and under the vortex field, the DEM results match better with the experimental data. Further optimization in CFD and DEM simulations are necessary to modify the simulation results. According to the CFD simulation without consideration of turbulent particle dispersion and pressure gradient force $\left(\mathrm{F}_{\mathrm{P}}\right)$, there are no particles in the vortex field, which does not correspond to the reality. However by consideration of these two mentioned forces, the particles velocities in vortex field modeled by CFX simulations match very good with LDA results.

\section{HYDROABRASION}

The hydroabrasion of particulate flows is influenced by several important parameters including solid particles char- acteristics (concentration, impact velocity, impact angle, shape, hardness, density, size distribution), the fluid characteristics (flow rate, composition, density, viscosity), the component geometry (bend, choke, T-junction, pump, hydroturbine) and also the material properties (hardness, microstructure).

The characteristics of abrasive particles, component geometry and surface material properties are all given or defined for the boundary conditions of the simulations. Impingement information, such as impact velocity, impact angle and impact locations is collected as the abrasive particles hit the wall of the geometry through the flow field calculations. Using this information, the erosion rate of the surface of the obstacle inside the pipe can be predicted locally. The Eulerian-Lagrangian method is used to calculate the particle 


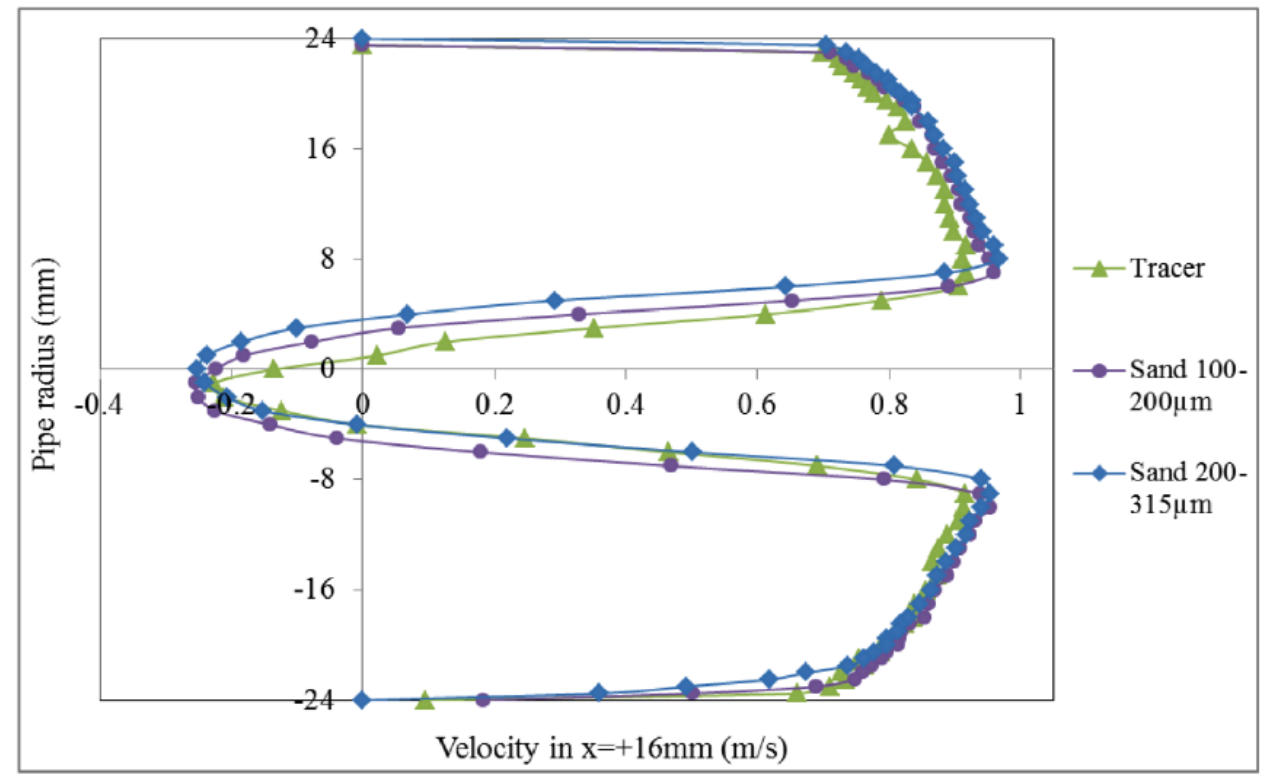

Fig. (9). Vel. profiles comparison among tracer and two size groups of sand particles.

trajectories and velocities. Normally for a defined mass of solid particles, the trajectories of tens of thousands of particles that are randomly distributed at the inlet are determined to obtain statistically representative solid particles impingements on the surface in order to acquire local erosion profiles over the surface of the geometry.

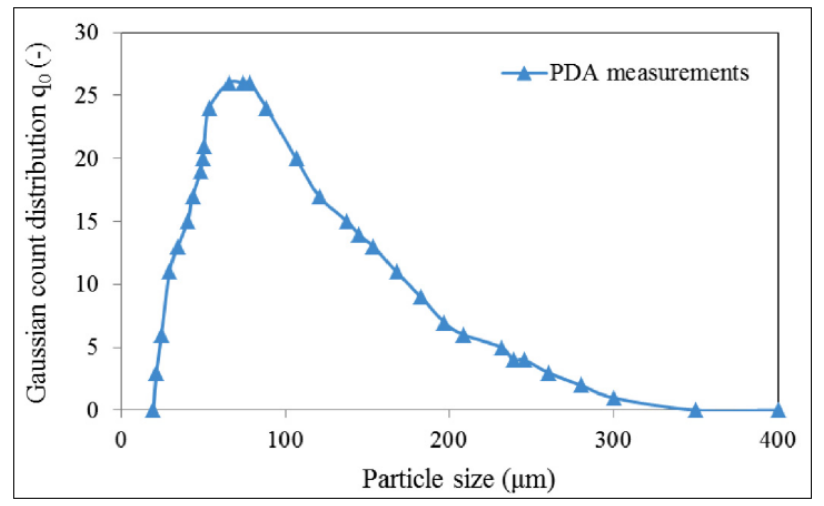

Fig. (10). Sand particles size distribution by PDA measurements.

There is no comprehensive formula currently capable of evaluating hydroabrasion quantitatively with consideration of all the influencing parameters. There is, however, a number of theoretical and experimental data enabling to address the erosion dependency from separate parameters under specific conditions. Ahlert and McLaury [18, 19] have experimentally indicated that the erosion of a specimen is mostly dependent on the particle impingement angle and its impact velocity. They defined the ratio of the eroded mass of target material to the mass of impinging solid particles as follows:

$$
E R=A F_{s} V^{1.73} f(\theta) B^{-0.59}
$$

where $F_{s}$ is the particle shape coefficient; here $F_{s}=1.0$ for sharp (angular), 0.53 for semi-rounded and 0.2 for fully rounded solid particles can be used, $\mathrm{V}$ is the local impact velocity, A is an empirical constant and B is the Brinell's hardness of the tested material. Alhert and McLaury [18] used two functional forms of the angle dependence, with matching conditions applied at $15^{\circ}$. The dependence on impingement angle, $f(\theta)$ is given by equations (14) and (15).

$$
\begin{aligned}
& f(\theta)=a \theta^{2}+b \theta \quad \text { for } \theta \leq 15^{\circ} \\
& \mathrm{f}(\theta)=\mathrm{x} \cos \theta^{2} \sin \theta+y \sin \theta^{2}+z \\
& \text { for } 90^{\circ}>\theta>15^{\circ}
\end{aligned}
$$

Overall erosion rate of the wall due to the solid particles interaction with the wall is finally computed from equation (16) where $\dot{N}$ is the number rate of solid particles and $m_{p}$ is the particle mean mass.

$$
\dot{E}=\dot{N} \cdot m_{p} \cdot E
$$

In the present study the erosion rate is given as erosion rate density derived by equation (16) with the unit of $\mathrm{kg} / \mathrm{s}$.

In general, the erosion rate can be also converted to the local wall thickness loss. The local wall thickness loss is defined as the local wall erosion (mass loss) rate divided by the local cell area and the density of the wall material. The erosion can be finally given as the penetration rate as length per mass unit, which represents the surface wall thickness loss per unit mass of abrasive particles loading and thereby the penetration rate is a solid particle-loading independent variable [20].

In (Fig. 12) it can be clearly observed that near the frontline of the cylindrical sample where the impact angle is near to $90^{\circ}$, no erosion or relatively very low erosion occurs. It is known that the flow momentum in the vicinity of cylinder stagnation point decreases. Moreover, according to the literature [21], the erosion of ductile material surfaces when the impact angle of the particulate flow is around $90^{\circ}$ is relatively small compared to the erosion by smaller angles where the cutting wear is the dominant erosion process. The erosion also decreases near the channel walls. With the help of trigonometric function, the maximum angle of erosion $\alpha_{\max }$ can be approximated. For this purpose the following equation is applied: 

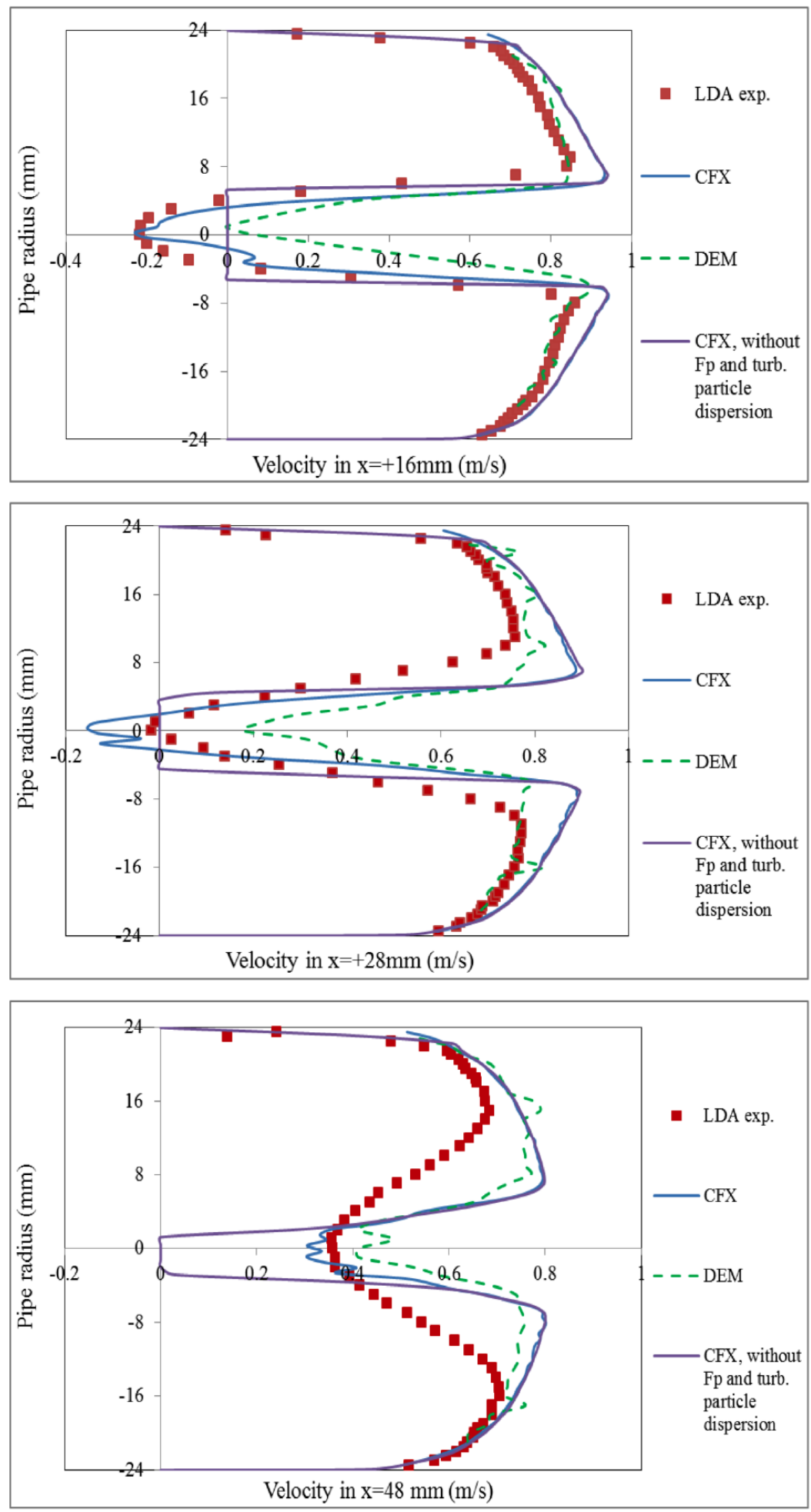

Fig. (11). DEM, CFD and LDA velocity profiles at 3 various positions behind the obstacle.

$$
\alpha_{\max }=90^{\circ}-\arcsin \left(\frac{y}{r}\right)
$$

where $r$ is the radius of the cylinder cross section and $y$ is the vertical distance between the maximum erosion location on the cylinder surface and the stagnation point of cylinder. According to the Fig. (12) by measuring the y, the $\alpha_{\max }$ was predicted graphically as $56.6^{\circ}$.
The erosion rate of the cylindrical obstacle made form stainless steel 1.4301 with the Vickers hardness of 235 HV30 was calculated as a function of sand particles concentration and feed volumetric flow and presented in (Fig. 13). A logarithmic increase in erosion rate by increase in feed volumetric flow is observed where the solid concentration is kept at 3 weight \% constant. It is also observed that the erosion rate increase linearly as a function of solid phase 


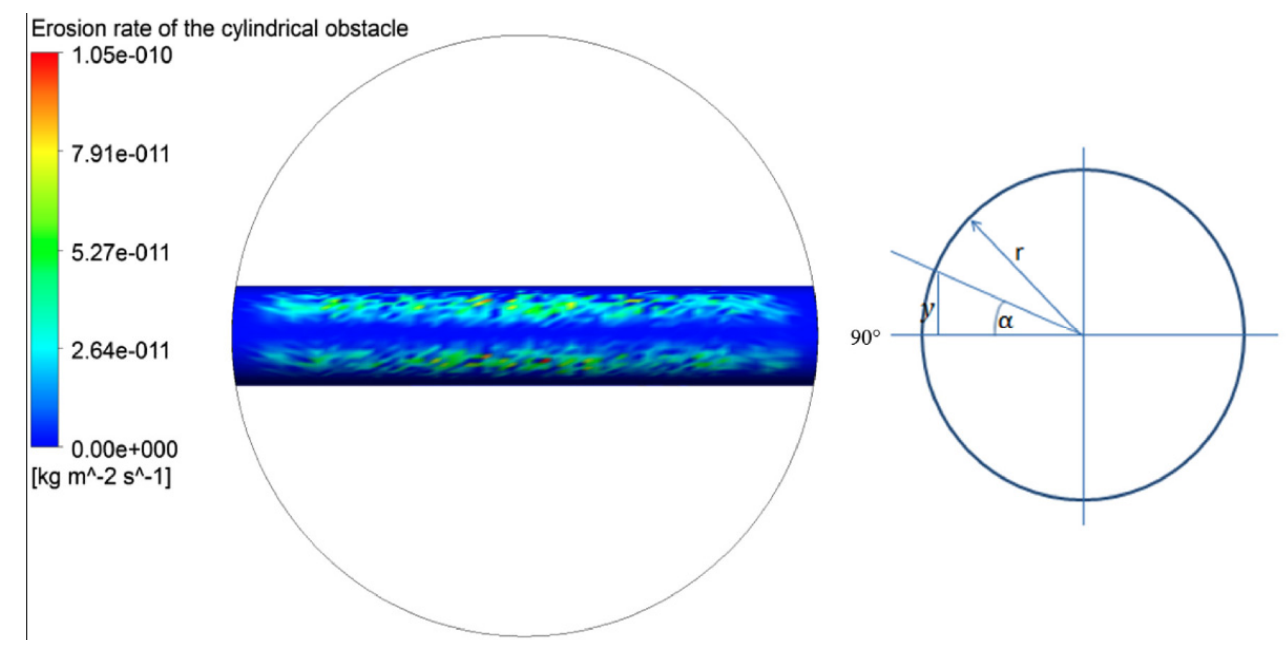

Fig. (12). Front view of erosion on the cylinder surface and predicting the maximum angle of erosion.

concentration where the volumetric feed flow is kept at 4000 $1 / \mathrm{h}$ constant. The location of erosion and the progress of erosion of the cylindrical obstacle surface as the weight concentration of solid particles increases is shown in (Fig. 14) with (a): $3 \%,(b): 5 \%$ and (c): $7 \%$ sand concentration.

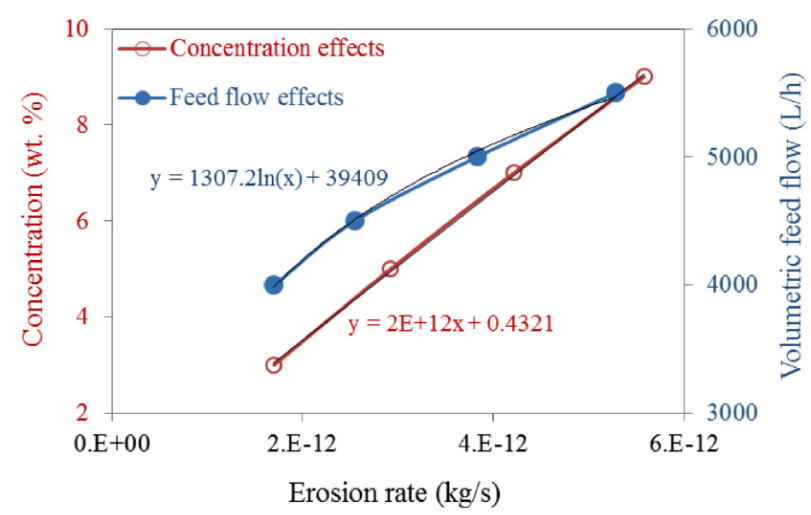

Fig. (13). Erosion rate of stainless steel versus feed flow and solid concentration.

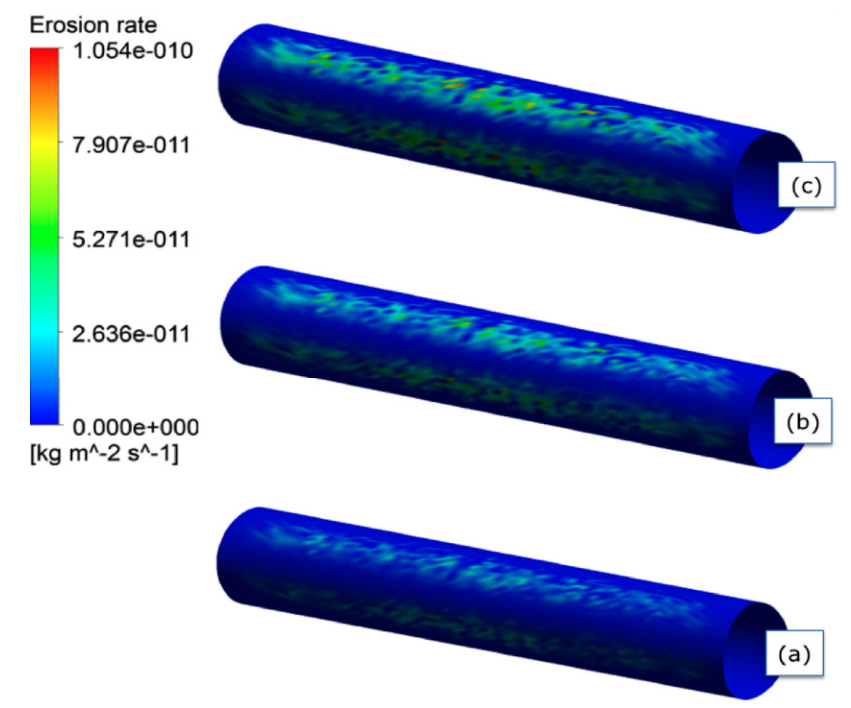

Fig. (14). Erosion of cylindrical obstacle by various sand concentrations.

\section{CONCLUSIONS}

In this work, an experimental tester was built and put into operation. The optical non-invasive method of Laser/Phase Doppler Anemometry was applied in order to investigate the flow behavior of particulate turbulent flow in a pipe channel. The flow inside the pipe is disturbed by the installation of a cylindrical obstacle and its influence was investigated by comparing the flow profiles in front and behind the obstacle in various defined positions. The measurement data are compared with the simulated CFD results. The motivation is to determine the flow behavior and effects on obstacles in pipelines with the help of LDA/PDA measuring technique and to provide a primary database for future hydroabrasion tests.

First, the turbulent single-phase flow by using tracer particles in water flow is characterized. Tracer particles have hardly any influence on the flow behavior of the fluid due to their small size and identical density as the water. The applied flow rate is linearly proportional to the measured velocity of the tracer particles. It was found that average velocities above the center of the tube are slightly larger than the lower half of the pipe cross section which coincides with the results of previous experiments. Behind the obstacle, the formation of a Karman vortex field is observed. According to the CFD simulations without consideration of turbulent particle dispersion and pressure gradient force, there are no solid particles in the vortex field, which does not correspond to reality. However by consideration of these two mentioned forces, the particles velocities in Karman vortex field match quite good with LDA results.

Furthermore, the particulate flow is investigated using sand particles having two different size distributions. With respect to the measured velocity profiles, there is a little variation when the particles are added. Compared to the single-phase flow, the sand particles have a slightly lower average velocity in the Karman vortex field and slightly larger velocity magnitudes above and under the Karman vortex field. The vortex field is also slightly wider, which is due to the increased shear forces of the particles. A comparison with the CFD results show that a higher average deviation of the velocity profile occurs as the flow travels away from the obstacle and redevelops itself. A comparison of CFD and 
DEM results with experimental data showed that in Karman velocity field behind the obstacle the CFD results fit better to the LDA measurements. In the fully developed flow region and also above and under the vortex field behind the obstacle, the DEM results match better with the experimental LDA data.

Hydroabrasion studies in the channel flow with higher amounts of solid particles were also performed and the increase in erosion of obstacle by increase in feed volumetric flow and solid particles concentration is observed. Furthermore, the maximum angle of erosion was calculated as $56.6^{\circ}$ using trigonometric function by assuming the $90^{\circ}$ angle where the flow hits the cylinder stagnation point.

\section{CONFLICT OF INTEREST}

The author(s) confirm that this article content has no conflicts of interest.

\section{ACKNOWLEDGEMENTS}

The authors would like to thank "Stiftung RheinlandPfalz für Innovation” for financial support.

\section{REFERENCES}

[1] M. Sommerfeld, "Analysis of collisions effects for turbulent gasparticle flow in a horizontal channel: Part I. Particle transport", Int. J. Multiph. Flow, vol. 29, pp. 675-699, 2003.

[2] N. Huber, M. Sommerfeld, "Characterization of the cross-sectional particle concentration distribution in pneumatic conveying systems", Powder Technol., vol. 79, pp. 191-210, 1994.

[3] Y. Tsuji, Y. Morikawa, "LDV measurements of an air-solid twophase flow in a horizontal pipe", J. Fluid Mech., vol. 120, pp. 385409, 1982.

[4] M. Ozgoren, E. Pinar, B. Sahin, H. Akilli, "Comparison of flow structures in the downstream region of a cylinder and sphere", Int. J. Heat. Fluid Flow, vol. 32, pp. 1138-1146, 2011.

[5] W.A.S. Kumara, G. Elseth, B.M. Halvorsen, M.C. Melaaen, "Comparison of Particle Image Velocimetry and Laser Doppler Anemometry measurement methods applied to the oil-water flow in horizontal pipe", Flow Measurement and Instrumentation, vol. 21, pp. 105-117, 2010.
[6] S. S. Ristic, J. T. Ilic, D. S. Cantrak, O. R. Ristic, N. Z. Jankovic, "Estimation of Laser-Doppler Anemometry measuring volume displacement in cylindrical pipe flow", Thermal. Sci., vol. 16, No. 4 pp. 1027-1042, 2012.

[7] F. Durst, H. Kikura, I. Lekakis, J. Jovanovic, Q. Ye, "Wall shear stress determination from near-wall mean velocity data in turbulent pipe and channel flows", Exp Fluids, vol. 20, pp. 417-428, 1996.

[8] P.A. Langston, M.A. Al-Awamleh, F.Y. Fraige, B.N. Asmar, "Distinct element modeling of non-spherical frictionless particle flow", Chem. Eng. Sci., vol. 59, pp. 425-435, 2004.

[9] H.P. Zhu, Z.Y. Zhou, R.Y. Yang, A.B. Yu, "Discrete particle simulation of particulate systems: Theoretical developments", Chem. Eng. Sci., vol. 62, pp. 3378-3396, 2007.

[10] B.N. Asmar, P.A. Langston, A.J. Matchett, J.K. Walters, "Validation tests on a distinct element model of vibrating cohesive particle systems", Comp. Chem Eng., vol. 26, pp. 785-802, 2002.

[11] S. Radl, E. Kalvoda, B. J. Glasser, J. G. Khinast, "Mixing characteristics of wet granular matter in a bladed mixer", Powder Technol., vol. 200, pp. 171189, 2010.

[12] R. Jackson, "Locally averaged equations of motion for a mixture of identical spherical particles and a newtonian fluid", Chem. Eng. Sci., vol. 52, pp. 2457-2469, 1997.

[13] S. Laín, M. Sommerfeld, "Euler/Lagrange computations of pneumatic conveying in a horizontal channel with different wall roughness", Powder Technol., vol. 184, pp. 76-88, 2008.

[14] P.G. Saffman, "The lift on a small sphere in a shear flow", J. Fluid Mech., vol. 22, pp. 385-400, 1965.

[15] R. Mei, "An approximate expression for the shear lift force on a spherical particle at finite Reynolds number", Int. J. Multiph. Flow, vol. 18, pp. 145-147, 1992.

[16] B. Oesterlé, T. Bui Dinh, "Experiments on the lift of a spinning sphere in a range of intermediate Reynolds numbers", Exp. Fluids, vol. 25, pp. 16-22, 1998.

[17] M. C. Roco, C. A. Shook, "Modeling of slurry flow: The effect of particle size", Can. J. Chem. Eng., vol. 61, pp. 494-503, 1983.

[18] K.R. Ahlert, "Effects of particle impingement angle and surface wetting on solid particle erosion on ANSI 1018 steel", M.Sc. thesis, mechanical engineering department, University of Tulsa, Tulsa, USA, 1994.

[19] X. Chen, B.S. McLaury, S.A. Shirazi, "Application and experimental validation of a computational fluid dynamics (CFD) based erosion prediction model in elbows and plugged tees", J. Comput. Fluids, vol. 33, pp. 1251-1272, 2004.

[20] X. Chen, B.S. McLaury, S.A. Shirazi, "A comprehensive procedure to estimate erosion in elbows for gas-liquid-sand multiphase flow", J. Energy. Res. Technol., vol. 128, pp. 70-78, 2006.

[21] R. G. Bayer, Mechanical wear prediction and prevention. Marcel Dekker, Inc., 1994.

(C) Azimian et al.; Licensee Bentham Open.

This is an open access article licensed under the terms of the Creative Commons Attribution Non-Commercial License (http://creativecommons.org/licenses/by-nc/3.0/) which permits unrestricted, non-commercial use, distribution and reproduction in any medium, provided the work is properly cited. 